\title{
New Synthesis of Heterocycles via Azatitanacyclopentadiene Intermediates
}

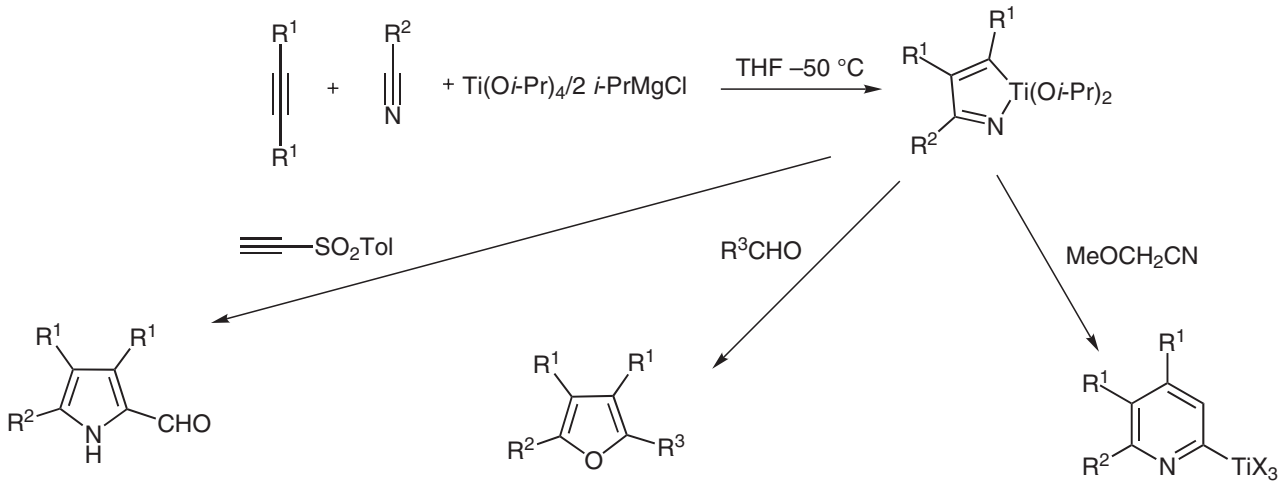

Significance: The reaction between acetylenes and nitriles in the presence of titanium(II) alkoxides represents an easy route for the preparation of various polyfunctional heterocycles. A number of substituted pyridines, pyrroles and furans, bearing a variety of substituents, were prepared under mild conditions in good yields. A very operationally simple procedure was developed.
Comment: Although titanacyclopentadienes have been successfully used in the synthesis of heterocycles, titanazacyclopentadienes were not known due to difficulties in their generation. Nevertheless, a number of functionalized titanacycles are available by a usual method, starting from cheap acetylenes, nitriles and divalent titanium species. This is an excellent method for the preparation of polysubstituted furans, pyridines and pyrroles, not easily accessible by other routes. 